\title{
Empirical Analysis on the Effect of City Size Distribution on Regional Energy Efficiency
}

\author{
Shunyi Li \\ Institute of Urban Economics and Development, Guizhou University of Finance and \\ Economics, Guiyang 550025, China
}

\begin{abstract}
The characteristics of city size distribution can affect the regional energy efficiency. The empirical analysis based on the provincial panel data of China show that the regional energy efficiency is in an "inverted U" relationship with the concentration levels of city size distribution. The study demonstrates that the energy efficiency optimization levels of well coordinated cities are higher than those overly dispersed or over-concentrated cities. It follows that the higher the urbanization rate, the stronger the "inverted U" effects on the energy efficiency would be produced. Hence, the urbanization path of coordinated development of cities at different scales is the optimization choice to improve regional energy efficiency.
\end{abstract}

\section{Keywords}

City Size Distribution; Urbanization; Energy Efficiency

\section{城市规模分布对区域能源利用效率影响的 实证分析}

李顺毅

贵州财经大学贵州城镇经济与发展研究院, 贵阳 550025, 中国

摘要：一个区域内的城市规模分布特征会影响该区域的能源利用效率。基于中国省际面板数 据的实证分析表明：在城市体系中，随着城市规模分布的集中度逐渐提高，区域能源利用效 率呈现 “倒 U 型” 变化趋势。即相对于城市规模分布过度分散或过度集中的状态, 在大、 中、小城市分布相对协调的城市体系下，区域能源利用效率会更高。在城镇化进程中，城市 规模分布集中度与区域能源利用效率的 “倒 U 型” 关系还会随着城镇化率的提高被进一步强 化。由此说明, 不同规模城市协调发展的城镇化道路是提高区域能源利用效率的合理选择。

关键词：城市规模分布; 城镇化 ; 能源利用效率 


\section{1. 引言}

完善的城镇化战略不仅需要引导 农村人口进入城镇, 而且还需要合理 引导人口流动的方向, 即选择和塑造 合适的城镇体系规模结构。城市规模 分布是一定区域内人口规模不等的 大、中、小城市和小城镇的数量分布 状况, 它反映了城市体系的规模结构 特征。优化城市体系规模结构是我国 新型城镇化战略的重要内容。是否有 利于落实 “五大发展理念” 是检验城 镇体系规模结构是否合理的重要标 准。从绿色发展的视角来看, 改革开 放以来，我国的城镇化取得了巨大成 就, 与此同时, 我国的能源消费量也 在大幅度增加, 2015 年的能源消费总 量已经达到 1978 年的 7.5 倍多。城镇 化是推动能源消费增加的重要因素

（刘耀涁，2007），与发达国际相 比, 中国城镇化进程中能源消费的增 长速度更快。城镇化率从 $39.6 \%$ 提高到 $50.5 \%$ 的阶段中, 美国人均能源消费增 加速度为每年 0.062 吨标准煤, 中国为 0.164 吨标准煤; 而且, 在大致这个发 展阶段上, 中国的人均能源消费增速 也高于日本和韩国（刘江华等, 2015 ）。面对能源高消耗带来的资源 环境约束, 在新型城镇化进程中塑造 怎样的城镇规模分布结构更有利于提 升能源利用效率就成为一个十分值得 关注的问题。分析和检验城镇化进程 中区域城镇规模分布结构与能源利用 效率之间的关系，不仅可以丰富我们 对能源利用效率影响因素的认识, 而 且对于制定有利于提高能源利用效率 的城镇化发展战略具有重要意义。

\section{2. 理论分析与研究假说}

在讨论城市规模分布结构对区域 能源利用效率的影响时, 本文首先分
析单个城市的规模与能源利用效率的 关系, 然后在此基础上分析城市体系 的规模分布特征对区域能源利用效率 产生的影响。

从单个城市的规模与能源利用效 率的关系来看，城市规模不同产生的 集聚效应和规模收益存在差异, 从而 影响能源利用的效率。不同规模的城 市具有不同程度的聚集效应和外部成 本, 前者边际收益递减, 后者则边际 成本递增, 因此存在一个净规模收益 最大的最优城镇规模, 城镇的净规模 效应首先随着城镇规模扩大而上升, 在达到峰值之后缓慢下降, 即城市规 模的大小与净规模收益之间呈 “倒 U 型” 变化（王小鲁、夏小林，1999;

Au \&Henderson，2006）。一方面, 城 市规模扩大可以产生明显的集聚效 应, 通过知识和技术的外溢、投入品 分享、服务专业化、要素市场的成 熟、基础设施共享等途径带来正外部 性, 集聚带来的正外部性会促进当地 全要素生产率的提高, 而能源作为一 种投入要素, 能源效率也会随者全要 素生产率的提高而提高（王海宁、陈 媛媛，2010）。另一方面，城市规模 也不是越大越好, 过度集聚会带来高 昂的成本（Henderson，2002），当城 市规模膨胀到一定程度后, 城市的管 理成本、交通拥挤成本、城市扩容的 建设成本等不断攀升, 而生产效率的 提升会放缓甚至下降, 出现低效集聚 和规模收益递减问题, 从而降低能源 利用效率。一些经验研究也支持了上 述分析。周圣强和朱卫平 (2013) 的 研究表明随着经济集聚度的提升, 就 业密度、企业密度的拥挤效应越来越 凸显, 经济效率与集聚度存在着 “倒 $\mathrm{U}$ 型” 关系。李静等 (2014) 的研究 也表明城市规模与全要素生产率存在 “倒 U 型” 关系。唐根年等 (2009) 的实证研究也发现在空间聚集过度并 呈现生产要素拥挤的情况下, 会出现 
生产要素的输入过剩和输出效率损 失。而段文斌和余泳泽 (2011) 的研 究则进一步表明全要素生产率增长与 提升我国能源效率具有一致性。因 此, 城镇规模与能源利用效率之间具 有 “倒 U 型” 关系, 即在城市规模扩 大过程中, 能源利用效率先会提升, 但当规模扩大到一定程度后能源效率 则会转而下降。

对于城市体系中城市规模的分布 特征, 可以大致分为三种类型：一是 分散型结构, 即大城市规模不足, 而 中小城市和小城镇数量较多; 二是协 调型结构，这种类型的城市体系中， 大、中、小城市和小城镇的规模和数 量适当, 形成较协调的金字塔型层次 结构; 三是集中型结构, 其中处于城 市体系顶端的大城市规模偏大, 人口 向大城市集中, 中小城市和小城镇普 遍规模偏小, 各级城镇间的规模差距 较大。从不同规模分布特征的城市体 系对区域能源利用效率产生的影响来 看, 在分散型城市体系中, 与协调型 结构相比, 一方面, 大城市规模不 足, 其能源使用的规模效益不能充分 发挥出来; 而中小城市由于聚集程度 低, 能源效率也较低。由此造成分散 型城市体系的总体能源效率比协调型 城市体系要低。对于集中型城市体系 来说, 当集中度达到一定程度后, 规 模过大的顶层城市会出现拥挤效应和 规模收益递减从而使能源利用效率下 降; 同时, 这种体系中城镇规模差距 很大, 除过规模大的顶层城市外, 其 余大量的城镇规模偏小, 难以发挥规 模效益, 导致这些城镇的能源效率效 益较低。因此, 集中型城市体系的总 体能源效率与协调型城市体系相比也 是偏低的。综上所述, 与过度分散或 过度集中的城市体系相比, 在城市规 模分布相对协调的城市体系中区域能 源利用效率更高。由此提出假说 1 : 在 城市体系中, 城市规模分布的集中程
度与区域能源利用效率呈现 “倒 U 型”关系。

进一步考虑在城镇化进程中随着 城镇化率的提高, 城市规模分布结构 对区域能源利用效率的影响会发生怎 样的变化。在城镇化率提高过程中农 村进城人口的流向会受到城市体系结 构的影响。如果在不改变城市规模分 布特征的情况下推进城镇化, 人口城 镇化率的提高会进一步固化原有的城 市规模分布结构。这种城市规模分布 特征的固化也会促使其对区域能源利 用效率的影响作用在原有基础上更为 突出。由此可以提出假说 2: 在不改变 城市体系结构特征的情况下, 城市规 模分布集中度与区域能源利用效率的 “倒 U 型” 关系会在城镇化率提升的 过程中被进一步强化。

\section{3. 计量模型与数据说明}

\section{1. 模型设定}

首先，对假说 1 进行检验。为了 检验城市规模分布与区域能源利用效 率的关系是否为 “倒 $U$ 型” , 本文在 回归模型中加入反映城市规模分布特 征变量的平方项, 而且考虑到当期的 能源效率可能会受到以往水平的影 响, 由此构建动态面板数据模型:

$$
\begin{aligned}
& E E_{i t}=\beta_{0}+\theta E E_{i t-1}+\beta_{1} u s s_{i t} \\
& +\beta_{2} u s s_{i t}{ }^{2}+\phi C_{i t}+\varepsilon_{i t}
\end{aligned}
$$

其中, 下标 $i$ 和 $t$ 表示样本的省份和年 份, $E E_{i t}$ 表示能源利用效率, $E E_{i t-1}$ 为能源利用效率的一阶滞后项, $u s s_{i t}$ 反映城市规模分布特征, $C_{i t}$ 为各控制 变量, $\varepsilon_{i t}$ 为误差项。

为了检验假说 2 , 本文借鉴 Rajan 和 Zingales(1998)提出的交互项方法, 构建如下模型: 
$E E_{i t}=\alpha_{0}+\lambda E E_{i t-1}+\alpha_{1} u r b a n_{i t} \times u s s_{i t}$

$+\alpha_{2} u_{r b a n} \times u_{i t} s_{i t}^{2}+\gamma C_{i t}+\varepsilon_{i t}$

其中, $\mathrm{urban}_{i t}$ 为城镇化率; $u_{r b a n} \times u_{i t} s_{i t}$ 和 $\alpha_{3} u_{r b a n} \times$ uss $_{i t}{ }^{2}$ 是城 镇化率与城市规模分布特征变量一次 项和二次项的交互项。根据 Rajan 和 Zingales ${ }^{[26]}$ 的方法, 如果模型 (2) 中 的估计系数 $\alpha_{1}$ 和 $\alpha_{2}$ 的符号与模型

(1) 中 $\beta_{1}$ 和 $\beta_{2}$ 的符号一致, 则表明 城镇化率提高会强化城镇规模分布集 中度与区域能源利用效率之间的 “倒 U 型”关系。

\section{2. 变量测算与数据来源}

本文中能源利用效率指标采用全 要素生产率框架下的能源效率, 其含 义是完成既定经济目标所需的最优能 源使用量与实际能源使用量之比 (原 毅军等，2012）。本文选择从全要素 生产率角度, 采用随机前沿模型测算 出的能源技术效率来度量能源效率。 在随机前沿生产函数的设定上, 本文 借鉴杨莉莉等 (2014) 的做法, 采用 投入要素的超越对数生产函数。产出 变量采用地区实际生产总值, 由各省 份名义 GDP 以 1978 年为基期使用居 民消费价格指数进行平减而得; 在投 入变量方面, 一是各省资本存量, 估 算方法采用单豪杰（2008）的永续盘 存法, 基期为 1978 年; 二是各省总就 业人数; 三是能源投入, 采用以万吨 标准煤为单位的各省能源消费总量度 量。本文使用 Fronitier4.1 软件进行估 计。本文中能源利用效率 $(E E)$ 即由 使用超越对数生产函数的随机前沿模 型得出的能源技术效率度量。

城市规模分布特征本文采用空间 基尼系数（ uss_gini）。本文借鉴 朱顺娟和郑伯红 (2014) 的方法, 计 算各省份的城市人口空间基尼系数, 公式如下:

$$
u s S \_ \text {gini }=\frac{1}{2(n-1) S} \sum_{i}^{n} \sum_{j}^{n}\left|p_{i}-p_{j}\right|
$$

空间基尼系数计算中，需根据人口数 量将城市从小到大排序，其中 $p_{i}$ 和 $p_{j}$ 表示第 $i$ 位城市和第 $j$ 位城市的人口数 量, $\mathrm{n}$ 为城市数量, $\mathrm{S}$ 为所有城市的总 人口。城市人口的空间基尼系数越大 表明城市规模分布越集中。

回归模型中的控制变量有: (1)经 济发展水平, 用各省的人均实际 GDP 对数 ( $L n g d p)$ 度量, 为获得实际 GDP 数据本文用居民消费价格指对名义 GDP 数据进行了平减处理。(2)产业结 构（str），用各省的第三产业增加值 在地区生产总值中所占比例来反映。(3) 城镇化率 (urban), 由各省城镇常住 人口占总人口比例计算。(4)对外开放 (trade), 由各省进出口总额与地区 生产总值之比来反映。(5)所有制结构 (soe)，由各省国有单位就业人数占 全省就业总人数的比重度量。

本文的样本为 2006-2011 年我国 22 个省级行政单位的面板数据, 数据 来自相关年份的《中国城市统计年 鉴》、《中国统计年鉴》、《中国能 源统计年鉴》、《中国国内生产总值 核算历史资料》和《新中国 60 年统计 资料汇编》。

\section{4. 估计结果}

对于动态面板数据模型的估计, 本文采用两步法系统 GMM 方法进行 估计。表 1 报告了运用两步法系统 GMM 方法对动态面板数据模型 (1) 和 (2) 进行估计的结果。各列估计结 果的 Sargan 检验均在 5\%的显著水平上 接受原假设, 说明工具变量是过度识 别; $\mathrm{AR}(1)$ 和 $\mathrm{AR}(2)$ 检验的结果说明在 $10 \%$ 的显著水平下存在一阶序列相关但 不存在二阶相关, 上述检验表明这里 使用估计方法是适用的。 
表 1 动态面板数据模型估计结果

\begin{tabular}{|c|c|c|}
\hline & $E E$ & $E E$ \\
\hline & (1) & (2) \\
\hline uss_gini & $\begin{array}{c}0.0049 * * * \\
(6.91)\end{array}$ & \\
\hline uss_gini ${ }^{2}$ & $\begin{array}{c}-0.0053 * * * \\
(-6.17)\end{array}$ & \\
\hline urban $\times$ uss_gini & & $\begin{array}{c}0.0277 * * * \\
(16.06)\end{array}$ \\
\hline$\underset{2}{u r b a n} \times{ }_{2}$ uss_gini & & $\begin{array}{c}-0.0364 * * * \\
(-13.71)\end{array}$ \\
\hline Lngdp & $\begin{array}{c}0.0001^{* *} \\
(2.48)\end{array}$ & $\begin{array}{c}-0.00004 \\
(-0.93) \\
\end{array}$ \\
\hline str & $\begin{array}{c}8.64 \mathrm{e}-06^{* * * *} \\
(2.91) \\
\end{array}$ & $\begin{array}{l}9.01 \mathrm{e}- \\
06 * * * \\
(2.64)\end{array}$ \\
\hline urban & $\begin{array}{l}0.0004 \\
(0.97)\end{array}$ & $\begin{array}{c}-0.0043 * * * \\
(-12.94)\end{array}$ \\
\hline trade & $\begin{array}{c}-0.0012 * * * \\
(-15.21)\end{array}$ & $\begin{array}{c}-0.0012 * * * \\
(-9.64)\end{array}$ \\
\hline soe & $\begin{array}{c}-0.0006 * * * \\
(-3.49)\end{array}$ & $\begin{array}{l}-0.0001 \\
(-0.76)\end{array}$ \\
\hline L.EE & $\begin{array}{c}0.3969^{* * * *} \\
(8.31)\end{array}$ & $\begin{array}{c}0.3383 * * * \\
(15.42)\end{array}$ \\
\hline 常数项 & $\begin{array}{c}0.0027 * * * \\
(6.98)\end{array}$ & $\begin{array}{c}0.0045 * * * \\
(15.89)\end{array}$ \\
\hline Sargan 检验 & $\begin{array}{c}16.682 \\
(\mathrm{p}=0.1017)\end{array}$ & $\begin{array}{c}15.129 \\
(\mathrm{p}=0.1278)\end{array}$ \\
\hline $\mathrm{AR}(1)$ 检验 $\mathrm{p}$ 值 & 0.032 & 0.055 \\
\hline $\mathrm{AR}(2)$ 检验 $\mathrm{p}$ 值 & 0.428 & 0.120 \\
\hline
\end{tabular}

注: ***、**和*分别表示 $1 \% 、 5 \%$ 和 $10 \%$ 的统计 显著水平上显著。回归系数对应的括号内为 $\mathrm{z}$ 统 计量。

从表 1 第 1 列的估计结果看，对 于区域能源利用效率，空间基尼系数 uss_gini 的一次项显著为正、二次项显 著为负。反映出城市规模分布的集中 程度与区域能源利用效率之间具有 “倒 U 型” 关系，验证了假说 1 。

在控制变量方面, 表 1 第 1 列 中, 人均实际 GDP 的系数显著为正, 说明经济发展与区域能源利用效率存 在正相关关系。产业结构的估计系数 显著为正，表明第三产业比重的提高 有利于提升区域能源利用效率, 这与 师傅和沈坤荣 (2013) 的研究结果是 一致的。城镇化率的估计系数为正,
但在统计上并不显著, 反映出样本时 期内我国城镇化进程并没有显著提升 能源利用效率, 这可能与以往我国城 镇化过程中高投入、高能耗的粗放式 增长方式有关。各省进出口总额占地 区生产总值比重的系数显著为负, 这 表明在样本时期内我国对外贸易并没 有起到提高能源利用效率的作用, 这 与杨莉莉等 (2014) 的实证结果是一 致, 原因可能是我国以加工贸易为 主, 出口的产品大多是技术含量相对 较低的传统产品, 增长模式粗放性问 题比较严重。所有制结构的估计系数 显著为负, 说明国有经济占比较高会 抑制能源利用效率提升，原因可能是 国有经济比重与产业绩效负相关（孙 早、王文，2010），能源作为投入要 素, 其效率也会受到国有企业绩效较 低的不利影响。此外, 能源利用效率 一阶滞后项的估计系数都显著为正， 说明当期能源利用效率的确受到之前 的影响, 是有必要设定动态面板数据 模型进行检验的。

从表 1 第 2 列的估计结果看, 城 镇化率与空间基尼系数一次项和二次 项的交互项估计系数结果分别是显著 为正和显著为负。与回归 (1) 中空间 基尼系没有做交互项的估计系数符号 是一致的。这说明在动态面板数据模 型下, 城镇化率的提高会进一步强化 城市规模分布集中度与能源利用效率 间 “倒 U 型” 关系, 验证了假说 2。

\section{5. 结论}

本文使用 2006-2011 年的我国 22 个省级行政单位的面板数据样本, 通 过动态面板数据模实证分析了城市规 模分布特征与区域能源利用效率之间 的关系, 以及城镇化率的提高会对上 述关系产生怎样的影响。结果表明: 在城市体系中, 城市规模分布的集中 度与区域能源利用效率之间存在 “倒 
U 型” 关系, 即相对于城市规模分布 过度分散或过度集中的结构, 在大、 中、小城镇分布相对协调的城市体系 下，区域能源利用效率会更高。而 且, 城市规模分布集中度对区域能源 利用效率的 “倒 U 型” 影响会随城镇 化率的提高被进一步强化。由此说 明, 在城镇化进程中, 不同规模层次 的城市协调发展的路径是有助于提高 区域能源利用效率的合理选择。

\section{致谢}

本研究得到了商务部联合基金项 目 “贵州绿色开放与新型工业化协调 发展研究” (2015SWBZD14)的资助。

\section{参考文献}

[1] 刘耀涁, 中国城市化与能源消费关 系的动态计量分析, 财经研究, 33 (11): 72-81,2007.

[2] 刘江华,邵帅,姜欣,城市化进程对能 源消费的影响：我们离世界水平还 有多远? - - 基于国内和国际数据 的比较考察, 财经研究, 41(2):111$122,2015$.

[3] 王小鲁,夏小林,优化城市规模 推动 经济增长, 经济研究,(9): 22-29,1999.

[4] C. C. Au and J. V. Henderson,Are Chinese cities too small, Review of Economic Studies, 73(3): 549-576, 2006.

[5] 王海宁, 陈媛媛,产业集聚效应与工 业能源效率研究——基于中国 25 个工业行业的实证分析, 财经研究, 36(9): 69-79,2010.

[6] J. V. Henderson, Urban primacy, external costs and quality of life, Resource and Energy Economics, 24(1-2): 95-106,2002.

[7] 周圣强, 朱卫平,产业集聚一定能带 来经济效率吗: 规模效应与拥挤效 应,产业经济研究, (3): 12-22,2013.
[8] 李静, 黄丹丹, 彭翡翠, 城市规模与 企业全要素生产率关系的实证研 究, 合肥工业大学学报(社会科学 版),28(5): 19-25,2014.

[9] 唐根年, 管志伟, 秦辉,过度集聚、 效率损失与生产要素合理配置研 究,经济学家, (11): 52-59,2009.

[10]段文斌, 余泳泽, 全要素生产率增长 有利于提升我国能源效率吗?- 基于 35 个工业行业面板数据的实 证研究, 产业经济研究, (4):78-88, 2011.

[11]R.Rajan and L.Zingales, Financial dependence and growth,American Economic Review, 88(3): 559-586, 1998.

[12] 原毅军, 郭丽丽, 孙佳, 结构、技 术、管理与能源利用效率——基于 2000-2010 年中国省际面板数据的 分析, 中国工业经济, (7): 18-30,2012.

[13] 杨莉莉, 邵帅, 曹建华, 任佳, 长三角 城市群工业全要素能源效率变动分 解及影响因 素——基于随机前沿 生产函数的经验研究, 上海财经大 学学报, 16(3):95-102,2014.

[14]单豪杰，中国资本存量 K 的再估 算: 1952-2006 年,数量经济技术经 济研究,(10): 17-31,2008.

[15]朱顺娟, 郑伯红,从基尼系数看中国 城市规模分布的区域差异, 统计与 决策, (8): 127-129,2015.

[16]师傅, 沈坤荣,政府干预、经济集聚 与能源效率, 管理世界, (10): 6-18, 2013.

[17] 孙早, 王文, 产业所有制结构变化对 产业绩效的影响一一来自中国工业 的经验证据,管理世界, (8): 66-78, 2011. 